\title{
Editorial
}

\section{Addressing oral cancer treatment in COVID 19 pandemic: The Indian scenario}

\author{
Ketki Kalele $^{1, *}$, Harshal Basatwar ${ }^{2}$ \\ ${ }^{1}$ Dept. of Oral Pathology, V.Y.W.S. Dental College \& Hospital, Amravati, Maharashtra, India \\ ${ }^{2}$ SMBT Dental College \& Hospital, Sangamner, Maharashtra, India
}

\section{A R T I C L E I N F O}

Article history:

Received 05-07-2020

Accepted 07-07-2020

Available online 18-07-2020
(C) 2020 Published by Innovative Publication. This is an open access article under the CC BY-NC license (https://creativecommons.org/licenses/by-nc/4.0/)
The outbreak of COVID-19 pandemic that initiated in Wuhan, China in December 2019, is a well known health emergency of today. The pandemic has affected millions of people across 204 countries. ${ }^{1}$ This highly infectious virus is not only taking toll on our day to day life and finances but also is severely affecting the much needed patient treatment and care for other gravid diseases including cancer. Especially, in developing countries like India which reports huge number of oral cancer cases \& wherein the available resources for treatment are also limited, the condition becomes all the more worrisome. ${ }^{2,3}$

Moreover, neglection on the treatment of patients with cancer can cause remarkable mortality \& morbidity as also ${ }^{3}$ timely treatment delivery to especially to stage 1 cancer patients is moral and ethical as the cure rates are high in these cases. ${ }^{4}$ On the other hand, hospital admission of cancer patients is a concern as these cases are immunodeficient \& are highly prone for COVID19 infections. ${ }^{5}$ In addition in Indian scenario wherein thousands of cancer patients are awaited for their treatment the hospital settings are also limited.

We, being the healthcare operators have to come up with a protocol for oral cancer patient management. This should be done by taking into consideration the treatment needs of individual oral cancer patient \& the state of the epidemic in the particular region that too in the resource

\footnotetext{
* Corresponding author.

E-mail address: drketkikalele@gmail.com (K. Kalele).
}

limited set up of our country. Proper designing of treatment guidelines for such patients should aim to protect oral cancer patients without causing much compromise on their oncologic outcome. ${ }^{6}$ This can be achieved by framing a multidisciplinary team (MDT) of surgeons, physicians, radiologists, radiotherapists, pathologists, hospital administrators, caregivers, counselors as well as members of the epidemic prevention board is the need of an hour. ${ }^{5,6}$

This team can have specific focus on area wise cancer patients \& the status of their specific treatment needs. Virtual meeting through videoconferencing \& through social media platforms can be encouraged \& special wards \& operation theaters should be prepared following strict sterilization protocols \& guidelines under the above mentioned MDT. ${ }^{5}$ The treatment strategy employed should consider anticancer therapy plus epidemic prevention. Some of the measures that can be employed are as follows:

1. Providing only palliative therapy for patients with advanced cancer which is potentially not curable or elderly patients $>70$ years with advanced disease; with the help of caregivers of the MDT. ${ }^{6}$

2. Strict rescheduling of the appointments of chemotherapy $\&$ radiotherapy so that not too many patients rush at the same time at the hospitals.

3. Filing of forms, registrations of patients as well as accountancy \& billing can be done online from the respective patients, which will minimize the amount of timing of the patients at the hospital. 
This can be monitored by the hospital administrators online $\&$ thus will reduce the number of personnel required in the hospital.

4. Definitive concomitant radiotherapy should be administered using simultaneous integrated boost technique (SIB) which can achieve 1 week reduction in the therapy than the standard therapy.

SIB also has benefits over tumor control \& prevention of toxicity due to prolonged therapy. ${ }^{6}$

5. Surgical interventions should not be done until \& unless the patient's thorough history including family history and travel history \& corona testing results are available.

This will not only prevent the patients from landing into complications but also will prevent the operating surgeons \& nurses from getting infected.

6. Last but not the least, proper training of the patient regarding COVID-19 including signs, symptoms as well as sterilization, proper hand washing techniques, other sanitization methods etc. to be given by MDT.

The above mentioned recommendations are aimed at providing a logical approach to treat the cancer patient in this extraordinary pandemic situation. The article is an attempt to shed light on the need of a proper MDT and designing of proper and specific treatment protocols for individual cancer patients.

\section{Conflict of Interest}

None.

\section{References}

1. Salari A, Shirkhoda M. COVID-19 pandemic \& head and neck cancer patients management: The role of virtual multidisciplinary team meetings. Oral Oncol. 2020;105:104693.

2. Jayaraj R, Poddar A, Aranha R, Royam M, Gothandam K, Nachimuthu R. Incidence, prevalence, and mortality associated with head and neck cancer in India: Protocol for a systematic review. Indian J Cancer. 2019;56(2):101-6.

3. Basu A, Ghosh D, Mandal B, Mukherjee P, Maji A. Barriers and explanatory mechanisms in diagnostic delay in four cancers - A healthcare disparity? South Asian J Cancer. 2019;8(4):221-5.

4. Bhatnagar S, Lohia N, Singh S, Prashar M, Subramananiam A, Viswanath S, et al. Survival trends in oral cavity cancer patients treated with surgery and adjuvant radiotherapy in a tertiary center of Northern India: Where do we stand compared to the developed world? SRM J Res Dent Sci . 2019;10(1):26-31.

5. Liang W, Guan W, Chen R. Cancer patients in SARS-CoV-2 infection: a nationwide analysis in China. Lancet Oncol. 2020;

6. Felice D. The impact of Coronavirus (COVID-19) on head and neck cancer patients' care. Radiother Oncol. 2020;147:84-5.

\section{Author biography}

Ketki Kalele Assistant Professor

Harshal Basatwar PG Student

Cite this article: Kalele K, Basatwar H. Addressing oral cancer treatment in COVID 19 pandemic: The Indian scenario. J Oral Med, Oral Surg, Oral Pathol, Oral Radiol 2020;6(2):41-42. 